\title{
Sensitivity analysis of Economic Value Added as a dominant indicator of concept of economic profit
}

\author{
Dusan Karpac ${ }^{1, *}$, Viera Bartosova ${ }^{1}$ \\ ${ }^{1}$ University of Zilina, Faculty of Operation and Economics of Transport and Communications, \\ Department of Economics, Univerzitna 1, 01026 Zilina, Slovakia
}

\begin{abstract}
Research background: The modern goal of enterprises, value creation, is achieved through the concept of economic profit. Profit, as part of profit or loss, is one of the most important flows, pointing to how efficiently corporate capital is used in an entity (Coatney \& Poliak, 2020). The article deals with the difference between accounting and economic profit, the selected form of economic profit - the EVA indicator. The economic value added (EVA) indicator is one of the best-known modern indicators of a company's performance (Siekelova et al., 2019). It shows whether the given entity increases its value or only earns for its economic survival. The benefit of this indicator is the valuation of equity and taking into account the risk. It is difficult to express the economic profit itself, therefore the article also addresses the issue of its calculation (Shah et al., 2016). The company needs to know its financial status and the direction it is heading, so we decided to calculate a selected form of economic profit.

Purpose of the article: The company needs to know its financial status and the direction it is heading, so we decided to calculate a selected form of economic profit. When expressing the value of the economic value added indicator, it is also important to know the items and components of the calculation that have the strongest meaning and effect on the possible amount of the indicator. Given this, we decided to use a sensitivity analysis, which points to the effect of individual variables that participate in the construction of the EVA calculation.

Methods: In this work, the methods of induction, deduction, and comparison were used to obtain a true picture of the subject issue. Methods of synthesis and analysis of the researched issues were also used.

Findings \& Value added: In the paper there is pointed out the intensity of the impact of individual variables that entered into the calculation of the economic value added indicator as a dominant indicator of concept of economic profit.
\end{abstract}

Keywords: Economic profit; Economic Value Added; Sensitivity Analysis

* Corresponding author: dusan.karpac@,fpedas.uniza.sk 
JEL Classification: $C 53 ; G 17 ; M 41$

\section{Introduction}

Naturally, every business unit wants to grow in the global market economy, increase its profits and expand its field of activity in order to grow its wealth from time immemorial (Fialova \& Folvarcna, 2020, Moorthi, 2021). The development of considering what is a priority for an entrepreneur in a business environment has gradually changed over the centuries and flexibly adapted to current global market conditions and opportunities. Initially, the subjects considered the most important increase in profit, which was reported in the accounting records of the entity, later in economic theory, the idea of increasing the value of the company was developed (Kliestik et al., 2020). Over time, businesses have realized that increasing accounting profit is not enough to sustain and grow in a market economy, and so the concept of economic profit has been added to the global corporate economy (Majercak et al., 2013).

The concept of economic profit consists of many indicators showing how efficiently a company can manage its assets, especially the indicator of economic value added is a very important apparatus of the concept (Jerabek et al., 2016). Given that economic profit is much more difficult to quantify than accounting profit, companies in the concept need a precise procedure for expressing this indicator (Shad \& Lai, 2015; Valaskova et al., 2020).

The assessment of the status of financial performance of business entities has shown a relatively dynamic development in the past. This trend has always been and still is a requirement for an information base on performance that changes in economic conditions (Shahid et al., 2017; Khan et al, 2020). The benefit of examining the financial status of entities is the generation of information about what individual companies tend to do in the financial direction in the future (Vochozka et al., 2016). The basis for analyzes of a financial nature are primarily financial statements, which are a tool used by the company's management to obtain relevant information (Savova, 2021; Siekelova et al., 2021).

The term sensitivity analysis is generally understood to mean the examination of the influence and changes of the input data on the values of the output data. The main goal of sensitivity analysis in our case is to get an overview of the effect of individual variables that participate in the construction of the calculation of a particular indicator. Some variables have an effect on the eventual value of the final indicator through the transfer of changes within several levels of the formula, other variables in the equation have a direct effect on the analyzed indicator.

\section{Methodology}

The basis for this study was the use of domestic and foreign professional literature, which was available in the form of scientific articles published in professional journals and conferences. Professional book publications and information from relevant websites were also used. The database necessary for the calculations related to the subject matter was based on data from balance sheets, profit and loss statements, and annexes to the financial statements of the selected company for the analyzed period 2015 - 2019. We chose a jointstock company that records its accounting according to accounting procedures in Slovak legislation. Based on the criteria, we chose a company whose data is transparent and publicly available. We will try to estimate the data that is not published by the company through a subjective perception based on a study of the available information about the company.

The sensitivity analysis was used in this paper to monitor the impact of possible changes in selected variables on the economic value added of the selected company, while it was processed using MS Excel. 


\section{Results}

Definitions of sensitivity analysis vary depending on its application in practice, but regardless of the differences in how sensitivity analysis is defined, it is always a process of examining how it is possible to quantify the effect of each input parameter on output variables (Pang et al., 2020). Salteli et al. (2008) argue that the three basic purposes of sensitivity analysis are factor prioritization, screening, and also mapping of factors involved in research. Other purposes may include the study of the interaction of individual parameters or the detection of errors occurring in the analyzed processes.

The main goal of sensitivity analysis in our case is to get an overview of the effect of individual variables that participate in the construction of the calculation of a particular indicator. Some variables affect the eventual value of the final indicator by transmitting changes within several levels of the formula, other variables have a direct effect on the analyzed indicator in the equation.

The year for the given analysis is 2019 , while we monitored the impact of selected variables in the case of their change by $-25 \%,-20 \%,-15 \%,-10 \%,-5 \%,+5 \%,+10 \%,+15 \%$ , $+20 \%$ and $+25 \%$. We monitored the possible impact of selected variables in relation to the indicator of economic value added and the captured changes are shown in the tables $1,2 \&$ 3.

In view of the tables, it can be said that the value of the EVA indicator of the selected company is most affected by variables such as net operating profit after tax, net operating assets, cost of equity, weighted average cost of capital and tangible fixed assets. It is also possible to demonstrate that the cost of equity has a significantly stronger effect on the final value of the economic profit of the selected company than the cost of depth capital. 


\begin{tabular}{|c|c|c|c|c|c|c|c|c|c|c|c|c|c|}
\hline \multirow{10}{*}{ 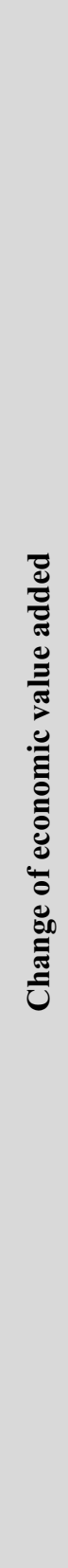 } & $\begin{array}{l}\stackrel{0}{0} \\
\stackrel{i}{+} \\
+\end{array}$ & $\begin{array}{l}\underset{g}{I} \\
\text { g } \\
\text { I }\end{array}$ & $\begin{array}{l}\stackrel{\circ}{\circ} \\
\text { مे } \\
\text { ते }\end{array}$ & 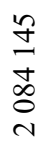 & 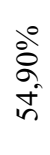 & $\begin{array}{l}\stackrel{m}{ \pm} \\
\stackrel{m}{\sigma}\end{array}$ & $\begin{array}{l}\text { ठें } \\
\text { ते } \\
\text { ते }\end{array}$ & $\begin{array}{l}\stackrel{2}{n} \\
\stackrel{\text { సे }}{-}\end{array}$ & $\frac{\partial}{\vec{\sigma}} \underset{i}{\stackrel{0}{r}}$ & $\begin{array}{l}\hat{0} \\
\infty \\
\infty \\
\alpha\end{array}$ & $\begin{array}{l}\stackrel{0}{\infty} \\
\stackrel{0}{0} \\
\stackrel{1}{1}\end{array}$ & 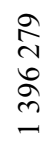 & $\frac{\stackrel{0}{\infty}}{\stackrel{\infty}{\infty}}$ \\
\hline & $\stackrel{\stackrel{\leftrightarrow}{+}}{\stackrel{T}{+}}$ & $\begin{array}{l}8 \\
\delta \\
\\
0 \\
-\end{array}$ & 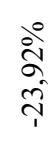 & $\begin{array}{l}\infty \\
0 \\
+ \\
0 \\
0 \\
\sigma \\
-1\end{array}$ & $\begin{array}{l}\stackrel{\circ}{\sigma} \\
\stackrel{\sigma}{\sigma}\end{array}$ & 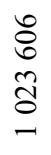 & $\begin{array}{l}\stackrel{\circ}{\alpha} \\
\text { } \\
\text { }\end{array}$ & $\begin{array}{l}0 \\
\text { 吕 } \\
\text { ô } \\
\text { ñ }\end{array}$ & $\begin{array}{l}\text { خे } \\
\text { ஸे } \\
\text { î }\end{array}$ & 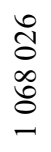 & $\begin{array}{l}\stackrel{0}{0} \\
\text { ర్} \\
\text { ণิ }\end{array}$ & 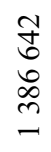 & 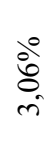 \\
\hline & $\frac{\stackrel{0}{i n}}{7}$ & $\begin{array}{l}? \\
0 \\
0 \\
0 \\
=\end{array}$ & $\begin{array}{l}\stackrel{0}{0} \\
\stackrel{+}{1} \\
\end{array}$ & $\begin{array}{l}\mathbb{N} \\
\infty \\
\infty \\
\infty \\
-\end{array}$ & $\begin{array}{l}\stackrel{0}{\circ} \\
\text { â } \\
\text { ते }\end{array}$ & 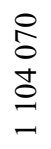 & $\frac{\stackrel{\circ}{+}}{\stackrel{+}{\sigma}}$ & $\begin{array}{l}0 \\
\delta \\
0 \\
\tilde{m}\end{array}$ & $\frac{\stackrel{0}{0}}{\stackrel{0}{i}}$ & $\begin{array}{l}\underset{D}{\infty} \\
\stackrel{n}{m} \\
\stackrel{-}{-}\end{array}$ & $\begin{array}{l}\stackrel{0}{\stackrel{0}{+}} \\
\stackrel{+}{6} \\
\stackrel{1}{1}\end{array}$ & $\begin{array}{l}\sqrt{n} \\
\frac{0}{n} \\
\frac{n}{2}\end{array}$ & $\begin{array}{l}\stackrel{0}{m} \\
\stackrel{2}{i}\end{array}$ \\
\hline & $\stackrel{\stackrel{0}{0}}{\underset{f}{+}}$ & $\begin{array}{l}\stackrel{+}{n} \\
n \\
\pm \\
\infty \\
=\end{array}$ & 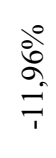 & 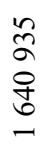 & 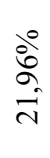 & $\begin{array}{l} \pm \\
\tilde{n} \\
\pm \\
\infty \\
-\end{array}$ & $\begin{array}{l}\stackrel{0}{0} \\
\stackrel{0}{1}\end{array}$ & 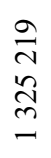 & $\begin{array}{l}\stackrel{0}{0} \\
\stackrel{n}{n} \\
i\end{array}$ & 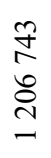 & $\begin{array}{l}\frac{0}{2} \\
\frac{0}{0} \\
\frac{0}{1}\end{array}$ & $\begin{array}{l}\infty \\
0 \\
i \\
0 \\
0 \\
0 \\
-1\end{array}$ & $\stackrel{\circ}{\stackrel{0}{n}}$ \\
\hline & $\stackrel{\circ}{i n}+$ & 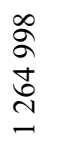 & $\begin{array}{l}\stackrel{0}{\circ} \\
\vdots \\
\hat{1}\end{array}$ & $\frac{\infty}{\stackrel{\infty}{\sigma}}$ & $\begin{array}{l}\stackrel{0}{0} \\
\stackrel{\circ}{0} \\
0\end{array}$ & 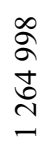 & $\begin{array}{l}\stackrel{0}{\circ} \\
\infty \\
\text { nิ }\end{array}$ & $\begin{array}{l}n \\
\infty \\
\sigma \\
\text { m } \\
m\end{array}$ & $\begin{array}{l}\stackrel{0}{\infty} \\
\stackrel{\infty}{0} \\
0\end{array}$ & 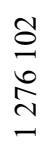 & $\frac{8}{80}$ & 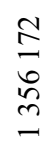 & $\begin{array}{l}\delta_{0}^{0} \\
\infty \\
0 \\
0\end{array}$ \\
\hline & in & 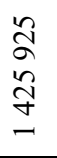 & 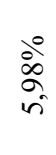 & $\begin{array}{l}\stackrel{2}{N} \\
\hat{a} \\
=\end{array}$ & $\begin{array}{l}0^{\circ} \\
\infty \\
0 \\
0 \\
1\end{array}$ & 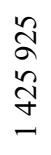 & $\begin{array}{l}\stackrel{0}{0} \\
\infty \\
\text { n. }\end{array}$ & $\begin{array}{l}\stackrel{\infty}{N} \\
b \\
\stackrel{0}{n} \\
-\end{array}$ & $\begin{array}{l}\stackrel{0}{\circ} \\
0_{0}^{\infty} \\
0\end{array}$ & $\begin{array}{l}\stackrel{\bigcirc}{\infty} \\
\infty \\
\searrow \\
\forall \\
-\end{array}$ & $\frac{\stackrel{0}{0}}{i n}$ & 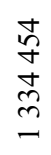 & $\begin{array}{l}\text { Oें } \\
\text { ó } \\
\text { i }\end{array}$ \\
\hline & $\stackrel{\circ}{\stackrel{0}{7}}$ & 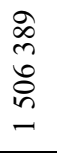 & $\begin{array}{l}\stackrel{0}{0} \\
\stackrel{0}{=}\end{array}$ & $\begin{array}{l}\infty \\
\infty \\
\curvearrowright \\
\stackrel{\sigma}{0} \\
-\end{array}$ & $\frac{\vec{\nabla}}{\vec{\sigma}}$ & 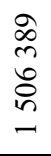 & $\begin{array}{l}\stackrel{0}{0} \\
\stackrel{0}{=}\end{array}$ & $\begin{array}{l}\infty \\
\infty \\
\infty \\
\infty \\
\infty \\
-\end{array}$ & $\stackrel{\stackrel{+}{+}}{\stackrel{0}{\tau}}$ & 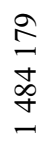 & $\begin{array}{l}\frac{0}{2} \\
\stackrel{2}{0} \\
\stackrel{0}{0}\end{array}$ & $\begin{array}{l}\infty \\
\stackrel{\infty}{n} \\
\underset{n}{\sim}\end{array}$ & $\underset{i}{\stackrel{0}{0}}$ \\
\hline & $\stackrel{\circ}{i}$ & 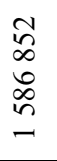 & 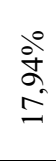 & 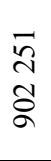 & $\begin{array}{l}\stackrel{0}{+} \\
\hat{\sigma} \\
\hat{\imath}\end{array}$ & $\begin{array}{l}\sim \\
\infty \\
\infty \\
\infty \\
\infty \\
-\end{array}$ & $\begin{array}{l}\stackrel{\circ}{+} \\
\stackrel{2}{\Xi}\end{array}$ & 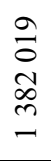 & $\stackrel{\stackrel{\circ}{N}}{i}$ & $\begin{array}{l}\infty \\
i n \\
n \\
n \\
n \\
-\end{array}$ & $\begin{array}{l}\stackrel{\circ}{\sigma} \\
\stackrel{5}{n}\end{array}$ & $\begin{array}{l}8 \\
i n \\
= \\
=\end{array}$ & $\begin{array}{l}\stackrel{\circ}{ } \\
\text { กิ } \\
\stackrel{1}{1}\end{array}$ \\
\hline & 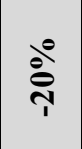 & 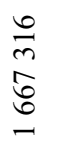 & $\begin{array}{l}\text { ڤे } \\
\text { సे }\end{array}$ & $\begin{array}{l}\vec{n} \\
\stackrel{n}{n} \\
\stackrel{+}{n}\end{array}$ & 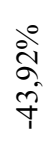 & $\begin{array}{l}0 \\
m \\
\hat{\sigma} \\
0\end{array}$ & $\begin{array}{l}\stackrel{0}{人} \\
\text { సे }\end{array}$ & 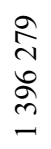 & $\frac{\stackrel{0}{\infty}}{\stackrel{\infty}{\infty}}$ & 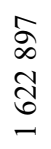 & $\begin{array}{l}\text { तें } \\
\text { ¿ें }\end{array}$ & 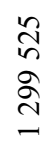 & $\frac{\partial 0}{\vec{F}}$ \\
\hline & 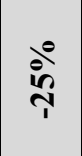 & 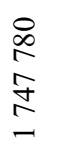 & $\begin{array}{l}\text { ठें } \\
\text { ڤे }\end{array}$ & $\begin{array}{l}\hat{2} \\
\hat{2} \\
\text { \& }\end{array}$ & $\begin{array}{l}\text { ठें } \\
\text { ڤे } \\
\text { î. }\end{array}$ & $\begin{array}{l}\stackrel{ }{\infty} \\
\stackrel{+}{+} \\
\underset{-}{\sim}\end{array}$ & $\begin{array}{l}\stackrel{\circ}{\circ} \\
\text { సू. }\end{array}$ & $\begin{array}{l}\stackrel{\circ}{\infty} \\
\Xi \\
\exists \\
\exists\end{array}$ & $\begin{array}{c}\stackrel{\circ}{\sigma} \\
\text { ๙े }\end{array}$ & 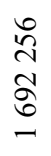 & 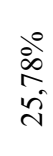 & $\begin{array}{l}\stackrel{8}{N} \\
\text { Do } \\
\text { N } \\
-\end{array}$ & $\begin{array}{l}\stackrel{\circ}{ } \\
\text { m } \\
\text { iे }\end{array}$ \\
\hline 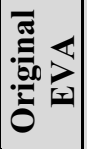 & $\stackrel{\circ}{\circ}$ & 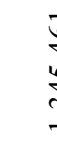 & f & & & & & & & & & & \\
\hline 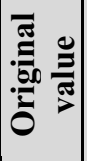 & $\stackrel{\circ}{\theta}$ & - & 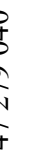 & & & & & & & & & & \\
\hline 产 & 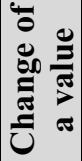 & & & & & & & & & & & & 흘 \\
\hline
\end{tabular}




\begin{tabular}{|c|c|c|c|c|c|c|c|c|c|c|c|c|c|}
\hline \multirow{10}{*}{ 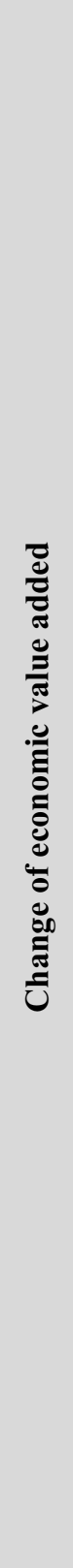 } & $\stackrel{\stackrel{0}{i}}{\stackrel{i}{+}}$ & $\begin{array}{l}\hat{n} \\
\hat{\alpha} \\
\stackrel{\infty}{-}\end{array}$ & $\begin{array}{l}\stackrel{0}{0} \\
\stackrel{+}{+}\end{array}$ & 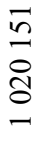 & $\begin{array}{l}\stackrel{0}{\infty} \\
\stackrel{+}{+} \\
\stackrel{+}{1}\end{array}$ & $\begin{array}{l}\vec{b} \\
+ \\
n \\
\stackrel{n}{5} \\
-1\end{array}$ & $\begin{array}{l}\stackrel{0}{8} \\
0_{0}^{\circ}\end{array}$ & 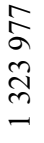 & $\frac{0}{8}{ }_{0}^{0}$ & \begin{tabular}{l}
0 \\
\multirow{2}{*}{} \\
๙े \\
ป
\end{tabular} & $\begin{array}{l}\stackrel{0}{0} \\
\infty \\
i \\
i\end{array}$ & \begin{tabular}{l}
$\vec{b}$ \\
+ \\
$n$ \\
\multirow{2}{*}{} \\
-
\end{tabular} & $\begin{array}{l}8 \\
8 \\
0\end{array}$ \\
\hline & $\underset{\stackrel{\sim}{+}}{\stackrel{\stackrel{\sim}{+}}{+}}$ & 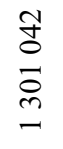 & $\begin{array}{c}\stackrel{0}{0} \\
\text { m} \\
\text { î }\end{array}$ & $\begin{array}{l}n \\
\sim \\
n \\
\infty \\
0 \\
-1\end{array}$ & $\begin{array}{l}\frac{0}{4} \\
\stackrel{2}{2} \\
\frac{1}{1}\end{array}$ & \begin{tabular}{l}
$\overrightarrow{5}$ \\
+ \\
$n$ \\
\multirow{2}{*}{} \\
-1
\end{tabular} & $\begin{array}{l}\stackrel{0}{8} \\
\text { ठ } \\
0\end{array}$ & 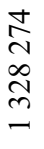 & $\begin{array}{l}\stackrel{0}{\circ} \\
\stackrel{\sim}{\rightarrow} \\
\rightarrow 1\end{array}$ & $\begin{array}{l}\text { ते } \\
\text { ठ } \\
\text { ㄱ. }\end{array}$ & $\begin{array}{l}0 \\
0 \\
\text { ల్ } \\
\text { }\end{array}$ & $\begin{array}{l}\vec{b} \\
\dot{+} \\
n \\
\tilde{y} \\
m\end{array}$ & $\begin{array}{l}80 \\
8 \\
0\end{array}$ \\
\hline & $\frac{\stackrel{\circ}{i}}{\text { in }}$ & 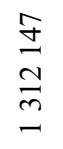 & $\begin{array}{l}\stackrel{0}{\circ} \\
\stackrel{+}{+} \\
i \\
i\end{array}$ & \begin{tabular}{l}
$n$ \\
\multirow{2}{n}{} \\
0 \\
$\stackrel{n}{n}$ \\
$=$
\end{tabular} & $\begin{array}{l}\frac{0}{n} \\
\frac{1}{1}\end{array}$ & \begin{tabular}{l}
$\overrightarrow{0}$ \\
+ \\
$n$ \\
\multirow{2}{*}{} \\
-
\end{tabular} & $\begin{array}{l}\dot{\theta}_{0} \\
\dot{0}^{\circ}\end{array}$ & $\begin{array}{l}\vec{n} \\
\sim \\
n \\
n\end{array}$ & $\begin{array}{l}0 \\
80 \\
0 \\
0 \\
0\end{array}$ & $\begin{array}{l}\stackrel{1}{a} \\
\stackrel{\infty}{\sim} \\
\end{array}$ & $\underset{\stackrel{i}{+}}{\stackrel{\leftrightarrow}{+}}$ & $\begin{array}{l}\vec{b} \\
+ \\
n \\
\stackrel{5}{m} \\
-\end{array}$ & $\begin{array}{l}0 \\
8 \\
0\end{array}$ \\
\hline & $\frac{\stackrel{\circ}{\stackrel{\theta}{+}}}{+}$ & $\begin{array}{l}\text { त̂ } \\
\text { ते } \\
\text { ñ } \\
-\end{array}$ & $\begin{array}{l}0 \\
b \\
b \\
-1\end{array}$ & $\begin{array}{l}\hat{m} \\
\hat{n} \\
\stackrel{n}{n}\end{array}$ & $\begin{array}{l}\stackrel{0}{\hat{b}} \\
a_{1}^{\circ}\end{array}$ & \begin{tabular}{l}
$\overrightarrow{5}$ \\
+ \\
$n$ \\
\multirow{2}{*}{} \\
\end{tabular} & $\begin{array}{l}\stackrel{0}{8} \\
\dot{0} \\
0\end{array}$ & $\begin{array}{l}\infty \\
0 \\
\infty \\
0 \\
0 \\
m \\
-\end{array}$ & $\begin{array}{l}\text { Oे } \\
\text { Oे } \\
0 \\
0\end{array}$ & $\begin{array}{l}\stackrel{n}{o} \\
\text { m } \\
\stackrel{n}{r}\end{array}$ & $\frac{i}{i n}$ & $\begin{array}{l}\vec{b} \\
+ \\
n \\
\dot{f} \\
m\end{array}$ & $\begin{array}{l}0 \\
8 \\
0\end{array}$ \\
\hline & 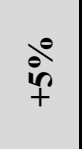 & 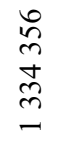 & $\begin{array}{l}0 \\
\infty \\
\infty \\
0 \\
i\end{array}$ & 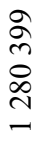 & $\begin{array}{l}\stackrel{\circ}{+} \\
\dot{+}_{1}\end{array}$ & \begin{tabular}{l}
$\overrightarrow{5}$ \\
+ \\
$n$ \\
\multirow{2}{*}{} \\
-1
\end{tabular} & $\begin{array}{l}\stackrel{\circ}{8} \\
\text { ठ } \\
0\end{array}$ & $\begin{array}{l}\exists \\
\Xi \\
\exists \\
\sim \\
=\end{array}$ & $\begin{array}{l}\stackrel{0}{2} \\
\text { ?े } \\
\text { iे }\end{array}$ & 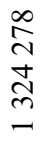 & $\frac{0}{\stackrel{0}{n}}$ & \begin{tabular}{l}
$\vec{b}$ \\
+ \\
$\vdots$ \\
\multirow{2}{0}{} \\
-
\end{tabular} & $\begin{array}{l}8 \\
8 \\
0\end{array}$ \\
\hline & in & $\begin{array}{l}8 \\
0 \\
n \\
0 \\
n \\
n \\
-\end{array}$ & $\begin{array}{l}0^{\circ} \\
\infty_{0}^{\infty} \\
0\end{array}$ & 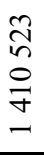 & $\begin{array}{l}\stackrel{0}{+} \\
\dot{+} \\
+\end{array}$ & $\begin{array}{l}\overrightarrow{5} \\
+ \\
n \\
\tilde{y} \\
m \\
-\end{array}$ & $\begin{array}{l}\stackrel{0}{8} \\
\stackrel{0}{0}\end{array}$ & 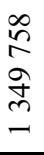 & 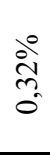 & $\begin{array}{l}\mathbb{J} \\
0 \\
0 \\
0 \\
0 \\
-\end{array}$ & $\stackrel{\circ}{\stackrel{0}{n}}$ & $\begin{array}{l}\vec{b} \\
+ \\
n \\
+ \\
- \\
-\end{array}$ & $\begin{array}{l}\circ \\
8 \\
0\end{array}$ \\
\hline & $\stackrel{\stackrel{0}{\ominus}}{\frac{1}{1}}$ & $\begin{array}{l}\vec{\sigma} \\
\hat{\sigma} \\
\pi\end{array}$ & $i_{-i}^{0}$ & $\begin{array}{l}n \\
\infty \\
n \\
n \\
\dot{y} \\
-\end{array}$ & $\begin{array}{l}\frac{0}{6} \\
a^{\circ}\end{array}$ & \begin{tabular}{l}
$\overrightarrow{5}$ \\
+ \\
$n$ \\
\multirow{2}{*}{} \\
\end{tabular} & $\begin{array}{l}\delta_{0}^{\circ} \\
\delta_{0}\end{array}$ & $\begin{array}{l}n \\
n \\
0 \\
\dot{0} \\
\text { ñ } \\
-\end{array}$ & $\begin{array}{l}0_{0}^{\circ} \\
0_{0}^{\circ}\end{array}$ & $\begin{array}{l}\hat{\lambda} \\
\infty \\
\hat{\infty} \\
\\
-\end{array}$ & $\frac{\stackrel{\circ}{i n}}{m}$ & \begin{tabular}{l}
$\vec{b}$ \\
+ \\
+ \\
\multirow{2}{0}{} \\
- \\
-
\end{tabular} & $\begin{array}{l}\circ \\
8 \\
0\end{array}$ \\
\hline & $\stackrel{\circ}{\frac{0}{T}}$ & $\begin{array}{l}\stackrel{2}{x} \\
\infty \\
\infty \\
\infty\end{array}$ & $\begin{array}{l}\stackrel{\circ}{\circ} \\
\stackrel{\infty}{+} \\
i\end{array}$ & $\begin{array}{l}\text { o } \\
\text { o } \\
0 \\
+ \\
n \\
-\end{array}$ & $\begin{array}{l}\frac{0}{\tilde{n}} \\
\dot{ \pm}\end{array}$ & \begin{tabular}{l}
$\overrightarrow{5}$ \\
+ \\
$n$ \\
\multirow{2}{*}{} \\
\end{tabular} & $\begin{array}{l}\stackrel{0}{8} \\
\text { ठ } \\
\text {. }\end{array}$ & $\begin{array}{l}\text { ñ } \\
\approx \\
\infty \\
n \\
n \\
-\end{array}$ & $\begin{array}{l}\dot{\Xi}_{0}^{\circ} \\
\text { o. }\end{array}$ & $\begin{array}{l}\stackrel{0}{0} \\
\text { a } \\
\text { oे } \\
\text { + }\end{array}$ & 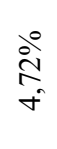 & \begin{tabular}{l}
$\vec{b}$ \\
+ \\
+ \\
\multirow{2}{0}{} \\
-
\end{tabular} & $\begin{array}{l}0 \\
8 \\
0\end{array}$ \\
\hline & 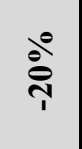 & 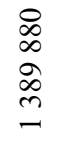 & $\begin{array}{l}\text { oे } \\
\text { nj }\end{array}$ & $\begin{array}{l}0 \\
\frac{1}{1} \\
n \\
0 \\
-1\end{array}$ & $\begin{array}{l}\stackrel{\circ}{+} \\
\stackrel{2}{2} \\
\stackrel{2}{2}\end{array}$ & \begin{tabular}{l}
$\overrightarrow{5}$ \\
+ \\
$n$ \\
\multirow{2}{*}{} \\
-1
\end{tabular} & $\begin{array}{l}\stackrel{\circ}{8} \\
\text { ठ } \\
0\end{array}$ & $\begin{array}{l}\text { aे } \\
\text { d } \\
\text { d } \\
\text { ? } \\
-\end{array}$ & $\begin{array}{l}\stackrel{0}{0} \\
\stackrel{1}{1} \\
-1\end{array}$ & 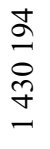 & $\begin{array}{l}\delta_{0}^{0} \\
\text { లn } \\
0_{0}\end{array}$ & \begin{tabular}{l}
$\vec{b}$ \\
+ \\
$n$ \\
\multirow{2}{*}{} \\
$n$ \\
-
\end{tabular} & $\begin{array}{l}\circ \\
8 \\
0\end{array}$ \\
\hline & 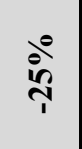 & \begin{tabular}{l}
$n$ \\
$\infty$ \\
0 \\
8 \\
\multirow{+}{+}{} \\
-1
\end{tabular} & $\stackrel{\stackrel{\circ}{7}}{\stackrel{+}{*}}$ & $\begin{array}{l}\stackrel{N}{\hat{N}} \\
\stackrel{0}{6} \\
-\end{array}$ & $\begin{array}{l}\frac{\infty^{0}}{ \pm} \\
\dot{d}\end{array}$ & $\begin{array}{l}\overrightarrow{5} \\
+ \\
n \\
\tilde{n} \\
\end{array}$ & $\begin{array}{l}\stackrel{0}{8} \\
\dot{\theta}_{0}\end{array}$ & 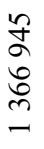 & $\stackrel{8}{8}$ & $\frac{n}{n}$ & $\begin{array}{l}\stackrel{0}{\circ} \\
\infty \\
i \\
\end{array}$ & $\begin{array}{l}\vec{b} \\
+ \\
n \\
\stackrel{5}{0} \\
-\end{array}$ & $\dot{8}_{0}^{\circ}$ \\
\hline 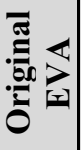 & $\stackrel{\circ}{\theta}$ & 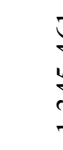 & & & & & & & & & & & \\
\hline 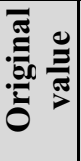 & $\stackrel{\circ}{\circ}$ & 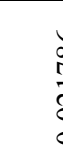 & & & & & & & & & & & \\
\hline 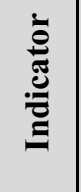 & 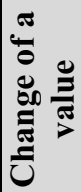 & & & & & & & & & & & & 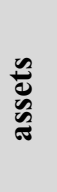 \\
\hline
\end{tabular}




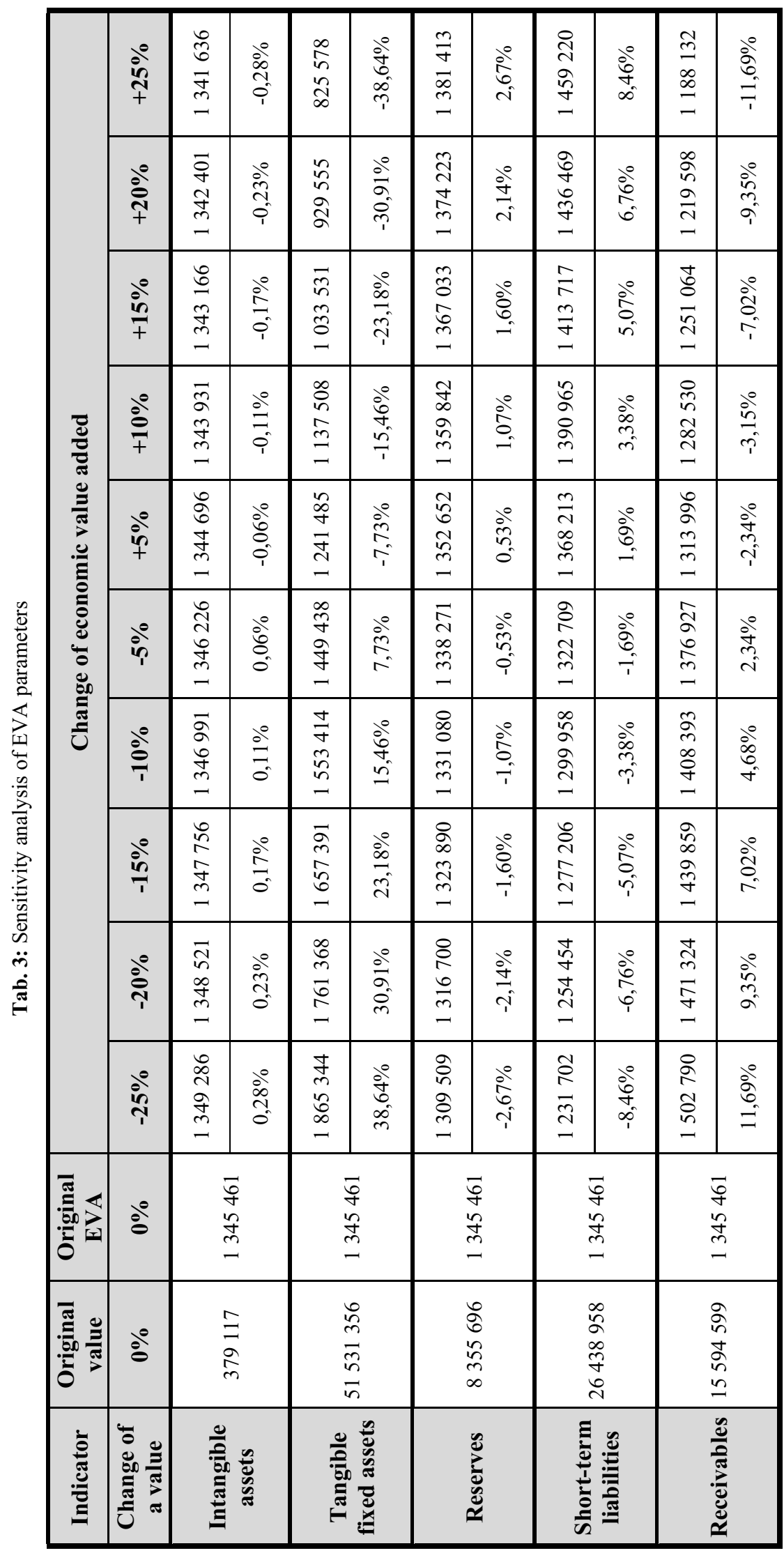




\section{Discussion}

Economic added value as a form of economic profit represents a part of the funds that will remain available to the business entity even after the payment of all its liabilities and capital costs.

Since the expression of economic added value of business entities requires many other calculations, included in its concept and based on various methods, which are not always unified, it is quite difficult and complicated to evaluate the indicator.

The paper deals with the sensitivity analysis, by which we pointed out the intensity of the impact of individual variables that entered into the calculation of the economic value added indicator. Sensitivity analysis basically represents the quantification of input parameters into output quantities, while the output in this context was the value of economic added value. The analysis showed that the most influential part of the EVA indicator is the net operating profit after tax, the effect of which on the potential value of economic value added is the strongest. Another finding from the analysis is that the effect of the cost of equity on the final amount of EVA is several times stronger than the effect that the cost of depth capital has on the value of EVA.

The data base necessary for the calculations of individual models and indicators related to the subject matter was based primarily on data from balance sheets, profit and loss statements and annexes to the financial statements of the selected company for the analyzed period 2015 - 2019. Choice of entity on which data were calculated practical part of the work, consisted in the criteria of a suitable legal form of business, transparent and sufficient accounting and especially the use of double-entry bookkeeping according to the procedures of Slovak accounting legislation. Due to this, we have chosen a joint-stock company that records its accounting according to the accounting procedures in Slovak legislation, while the data on the company are transparent and publicly available. We estimated the data that is not disclosed by the company through a subjective perception based on a study of the available information about the company.

\section{Conclusion}

Economic added value is a relatively important concept in the corporate economy in modern times, mainly because it can provide the owners of the company with information on how much the shareholder actually earns in the company or reports on profits in excess of paid liabilities and cost of capital (Robinson, 2020). This fact is really beneficial for business entities and the concept of economic profit, as well as its subconception of economic value added, still has a high potential to develop, both in economic-corporate theory and in the practice of business. Given that economic value added consists of many multi-level calculations, we decided to apply sensitivity analysis to its calculations to determine which parts and items are most significant in the EVA calculation. In view of the above, it can be said that the value of the EVA indicator of the selected company is most affected by variables such as net operating profit after tax, net operating assets, cost of equity, weighted average cost of capital and tangible fixed assets.

\section{Acknowledgements}

This is paper is an outcome of project VEGA: 1/0544/19 Formation of the methodological platform to measure and assess the effectiveness and financial status of non-profit organizations in the Slovak Republic. 


\section{References}

1. Coatney, K., \& Poliak, M. (2020). Cognitive Decision-Making Algorithms, Internet of Things Smart Devices, and Sustainable Organizational Performance in Industry 4.0based Manufacturing Systems. Journal of Self-Governance and Management Economics, 8(4), 9-18.

2. Fialova, V., \& Folvarcna, A. (2020). Default prediction using neural networks for enterprises from the post-soviet country. Ekonomicko-manazerske spektrum, 14 (1), 43 51.

3. Jerabek, K., Majercak, P., Kliestik, T., \& Valaskova, K. (2016). Application of Clark and Wright' s savings algorithm model to solve routing problem in supply logistics. Nase More, 63(3), 115-119.

4. Khan, K. A., Dankiewicz, R., Kliuchnikava, Y., \& Olah, J. (2020). How do entrepreneurs feel bankruptcy? International Journal of Entrepreneurial Knowledge, 8(1), 89-101.

5. Kliestik, T., Valaskova, K., Lazaroiu, G., Kovacova, M., \& Vrbka, J. (2020). Remaining Financially Healthy and Competitive: The Role of Financial Predictors. Journal of Competitiveness, 12(1), 74.

6. Majercak, P., Cisko S., \& Majercakova E. (2013). The impact of theory of constraints on the management accounting. In Proceedings of 7th International Days of Statistics and Economics (pp. 894-904).

7. Moorthi, S. B. S. (2021). Corporate performance measurement tool via economic value added. International Journal of Management, 8(3), 60-65

8. Pang, Z., O'Neil, Z., Li, Y., \& Niu, F. (2020). The role of sensitivity analysis in the building performance analysis: a critical review. Elsevier, 209, 109659.

9. Robinson, R. (2020). Computationally Networked Urbanism and Sensor-based Big Data Applications in Integrated Smart City Planning and Management. Geopolitics, History, and International Relations, 12(2), 44-50.

10. Salteli, A., Ratto, M., Andres, T., Campolongo, F., Cariboni, J., Gatelli, D., Saisana, M., \& Tarantola, S. (2008). Global sensitivity analysis: the primer. John Wiley \& Sons.

11. Savova, K. (2021). Variable application of accounting standards - current aspects, Ekonomicko-manazerske spektrum, 15(1), 111-122.

12. Shad, M., \& Lai, F-W. (2015). Conceptual framework for enterprise risk management performance measure through economic value added. Global business and management research: an international journal, 7(2), 11.

13. Shah, R., Haldar, A., \& Nageswara Rao S.V.D. (2016). Economic value added: Corporate performance measurement tool. Corporate Board role duties and composition, 11(1), 47-58.

14. Shahid, A., Irshad S., \& Shams Khakwani, M. (2017). A comparative study on Accounting Based Measures and Economic Based Measures to determine Shareholder Value Creation. Proceedings of the International Conference on Management, Business \& Techonology, 173-182.

15. Siekelova, A., Belas, J., Podhorska, I., Durana, P. (2021). Accrual-Based Earnings Management: A Case Study in V4 Focusing on Mining And Quarrying Sector. Acta Montanastica, Slovaca, 26(1), 70-83.

16. Siekelova, A., Kovacova, M., Adamko, \& P. Stehel, V. (2019). Profit management as an instrument for SMEs developing: the case for Slovakia. Marketing and Management of Innovations, 3, 285-296. 
17. Valaskova, K., Gavurova, B., Durana, P., \& Kovacova, M. (2020). Alter Ego Only Four Times? The Case Study of Business Profits in the Visegrad Group. E\&MEconomics and Management, 23(3), 101-119.

18. Vochozka, M., Rowland, Z., \& Vrbka, J. (2016). Financial analysis of an average transport company in the Czech Republic. Nase more, 63(3), 227-236. 